\title{
Criminologie
}

\section{Évaluation des effets de la télé-surveillance sur la criminalité commise dans 13 stations du métro de Montréal}

\section{Rachel Grandmaison et Pierre Tremblay}

Volume 30, numéro 1, printemps 1997

Criminalités économiques

URI : https://id.erudit.org/iderudit/017399ar

DOI : https://doi.org/10.7202/017399ar

Aller au sommaire du numéro

Éditeur(s)

Les Presses de l'Université de Montréal

ISSN

0316-0041 (imprimé)

1492-1367 (numérique)

Découvrir la revue

Citer cet article

Grandmaison, R. \& Tremblay, P. (1997). Évaluation des effets de la télé-surveillance sur la criminalité commise dans 13 stations du métro de Montréal. Criminologie, 30(1), 93-110. https://doi.org/10.7202/017399ar
Résumé de l'article

This paper provides an assessment of the impact ofCCTV cameras on the monthly incidence of crimes committed in Montreal's underground subway network. Since 13 of the 65 Montreal's underground stations were provided with such cameras (about 10 on average per target station) between August 1991 and January 1993, we examined the monthly volume of reported offenses in both target and control stations between January 1991 and December 1993. Our analyses have been unable to detect any overall impact of CCTV cameras on the behavior of offenders. And controlling for kinds of crime or for specific areas and places within subway stations (depending on the location of cameras) did not change or qualify the initial overall no-effect finding. 


\section{ÉVALUATION DES EFFETS DE LA TÉLÉ-SURVEILLANCE SUR LA CRIMINALITÉ COMMISE DANS 13 STATIONS DU MÉTRO DE MONTRÉAL ${ }^{2}$ Rachel Grandmaison (M.Sc.) ${ }^{2}$ et Pierre Tremblay ${ }^{3}$ (Ph.D.)}

This paper provides an assessment of the impact of CCTV cameras on the monthly incidence of crimes committed in Montreal's underground subway network. Since 13 of the 65 Montreal's underground stations were provided with such cameras (about 10 on average per target station) between August 1991 and January 1993, we examined the monthly volume of reported offenses in both target and control stations between January 1991 and December 1993. Our analyses have been unable to detect any overall impact of CCTV cameras on the behavior of offenders. And controlling for kinds of crime or for specific areas and places within subway stations (depending on the location of cameras) did not change or qualify the initial overall no-effect finding.

\section{INTRODUCTION}

Nous nous proposons dans cette étude d'évaluer l'impact préventif ou dissuasif des caméras de télévision installées, entre août 1991 et janvier 1993, dans 13 des 65 stations du métro de Montréal. Il est généralement admis que cette stratégie de prévention situationnelle est très souvent efficace (Cusson et coll., $1994:$ p. 17), y compris dans les réseaux de métro. Des études évaluatives ont été faites notamment pour le métro de Hong Kong (Gaylord et Galliher, 1991) et pour le métro de Londres (Burrows, 1980; Webb et Laycok, 1992) et les résultats sont encourageants. On pouvait donc s'attendre à ce que l'expérience-pilote réalisée par la Société de transport de la Communauté urbaine de Montréal produise également des effets bénéfiques. Malheureusement, ce n'est pas le cas, comme nous le verrons. L'objectif principal de cette étude, au-delà du constat d'échec auquel nos analyses nous conduisent, est de comprendre pourquoi la stratégie de la STCUM n'a pas donné les résultats escomptés.

L'étude est divisée en plusieurs sections. La première section analyse la sélection des stations de métro qui devaient faire partie de

1. Nous tenons à remercier, pour leur précieuse collaboration, MM. Gilbert Cordeau et André Progens du SPCUM, MM. Michel Dupuis, Fernand Patenaude et Roger Leduc ainsi que Mme Lucy Latulipe de la STCUM et, finalement, M. Bernard Noël.

2. Agente de recherche, École de criminologie, Université de Montréal.

3. Pierre Tremblay est professeur, École de criminologie, Université de Montréal, 3150, rue Jean-Brillant, H3T IN8. Tél. : 343-2046. Fax : 343-5650. 
l'expérience-pilote. La deuxième section propose une première évaluation globale de l'impact des caméras sur la criminalité dans les stations expérimentales. La troisième section examine leur impact différentiel sur diverses formes particulières de criminalité. La quatrième section examine la topographie de la criminalité et évalue l'impact des caméras selon les endroits où celle-ci se produit (quais, corridors, etc.). La dernière section évalue l'impact de la télé-surveillance dans la station de métro où le volume de criminalité est le plus élevé du circuit. En conclusion, nous montrons que les résultats décevants de cette expérience-pilote tiennent principalement à une planification inadéquate du projet de prévention lui-même (tel que défini dans un ouvrage récent du ministère de la Sécurité publique, Cusson et coll., 1994, p. 23).

\section{LE CHOIX DES STATIONS DE MÉTRO EXPÉRIMENTALES}

Il était raisonnable de supposer que les 13 stations de métro choisies pour l'expérience-pilote étaient celles qui présentaient un niveau de criminalité plus élevé que la moyenne. Nous avons donc examiné les statistiques criminelles disponibles et évalué le nombre de crimes rapportés à la police par station de métro pour la période de juillet 1991 à juin 1992. Bien entendu, tous les délits commis dans le métro ne sont pas nécessairement rapportés à la police, mais on peut penser que le pourcentage de délits non rapportés est relativement constant d'une année à l'autre ou d'une station de métro à l'autre. On sait également que les délits qui ne sont pas rapportés aux autorités policières sont de gravité moindre.

Comme le montre le tableau 1, l'incidence annuelle des crimes est en moyenne 2 à 3 fois moins élevée dans les "stations témoins" (celles qui n'ont pas été sélectionnées pour l'expérience) que dans les stations expérimentales (celles où les caméras de télévision furent installées).

\section{Tableau 1}

Incidence annuelle de la criminalité, par station. dans le métro de Montréal : stations expérimentales et stations témoins (juillet 1991 à juin 1992)

\begin{tabular}{lccc}
\hline \multirow{2}{*}{ Type de crimes } & \multicolumn{2}{c}{ INCIDENCE MOYENNE } & \multirow{2}{*}{ RATIO } \\
\cline { 2 - 3 } & $\begin{array}{c}\text { Stations } \\
\text { expérimentales* }\end{array}$ & $\begin{array}{c}\text { Stations } \\
\text { témoins* }\end{array}$ & \\
\hline Criminalité globale & 43,15 & 17,25 & 2,50 \\
Vols qualifiés & 5.54 & 4,06 & 1,36 \\
Agressions, voies de fait & 10,00 & 3,02 & 3,31 \\
Vols à la tire & 12,15 & 3,27 & 3,71 \\
\hline
\end{tabular}

* La liste des stations est présentée à l'Annexe 1. 
Dénombrer les délits en chiffres absolus peut être trompeur. Certaines stations ont un volume relativement élevé de délits mais connaissent également un achaladange plus élevé que d'autres. En tenant compte de l'achalandage et en calculant le taux annuel de délits rapportés par million de passagers (un passager qui utilise deux fois le métro est comptabilisé ici deux fois), on obtient des résultats similaires à ceux du tableau 1. Ainsi, le taux annuel de délits par station expérimentale est de 10,3, alors qu'il est de 6,2 par station témoin.

On pourrait donc en déduire que les stations de métro sélectionnées pour l'expérience-pilote furent bien «choisies». Une analyse plus détaillée indique, toutefois, que cette sélection des stations expérimentales laisse à désirer. En examinant le nombre de crimes commis entre juillet 1991 et juin 1992 par station de métro, nous constatons que seulement 7 des 13 stations les plus à risque en termes de criminalité furent choisies pour l'expériencepilote (cf. annexe 1). Lorsqu'on classe les stations de métro par ordre décroissant de taux de criminalité par million de passagers, seulement 4 des stations les plus à risque furent sélectionnées pour l'expérience-pilote (cf. annexe 1). Celle-ci avait ainsi pour objectif de prévenir la criminalité, mais cet objectif n'était ni le seul ni nécessairement le plus important. Nous reviendrons en conclusion sur les conséquences (souvent néfastes) pour un projet de prévention de poursuivre une variété d'objectifs plus ou moins compatibles.

\section{IMPACT GLOBAL DES CAMÉRAS DE TÉLÉVISION SUR LA CRIMINALITÉ DANS LES STATIONS EXPÉRIMENTALES}

Pour évaluer l'impact des caméras de télévision sur la criminalité dans les stations sélectionnées, notre plan ou devis évaluatif est de type « avantaprès ». Nous examinons l'évolution mensuelle de la criminalité avant l'installation des caméras et son évolution subséquente. La caméra elle-même est le stimulus expérimental. La période antérieure est appelée «période témoin » et la période subséquente, « période expérimentale ». Pour contourner le problème des variations saisonnières (baisse marquée des délits dans le métro durant la période estivale), nous examinons une série chronologique relativement longue. L'évaluation proposée est de nature quasi expérimentale, en ce sens que les stations expérimentales n'ont pas été choisies au hasard et qu'il est difficile de considérer les stations témoins et les stations expérimentales comme parfaitement comparables. Par ailleurs, l'évaluation de l'impact de l'expérience-pilote a été faite après coup (alors que dans les conditions idéales, cette évaluation est incorporée dès le début de l'expériencepilote et se poursuit tout au long de son déroulement). Bien qu'il s'agisse d'une évaluation quasi expérimentale, nous utilisons néanmoins, pour fins 
de commodité, les expressions de stations témoins et de stations expérimentales (sur la méthodologie quasi expérimentale, voir Cook et Campbell, 1979 ; Fortin, 1982 ; Cusson et coll., 1994, p. 48).

Comme le montre le graphique 1, l'installation des caméras dans les stations expérimentales s'est étalée sur une période d'environ un an : une première station fut équipée de caméras en septembre 1991, une deuxième, deux mois après. Les caméras des 11 autres stations expérimentales furent installées entre mai 1992 et janvier 1993.

\section{Graphique 1}

Incidence mensuelle de la criminalité rapportée à la police, dans le métro de Montréal : stations expérimentales et stations témoins (janvier 1991 à décembre 1993)

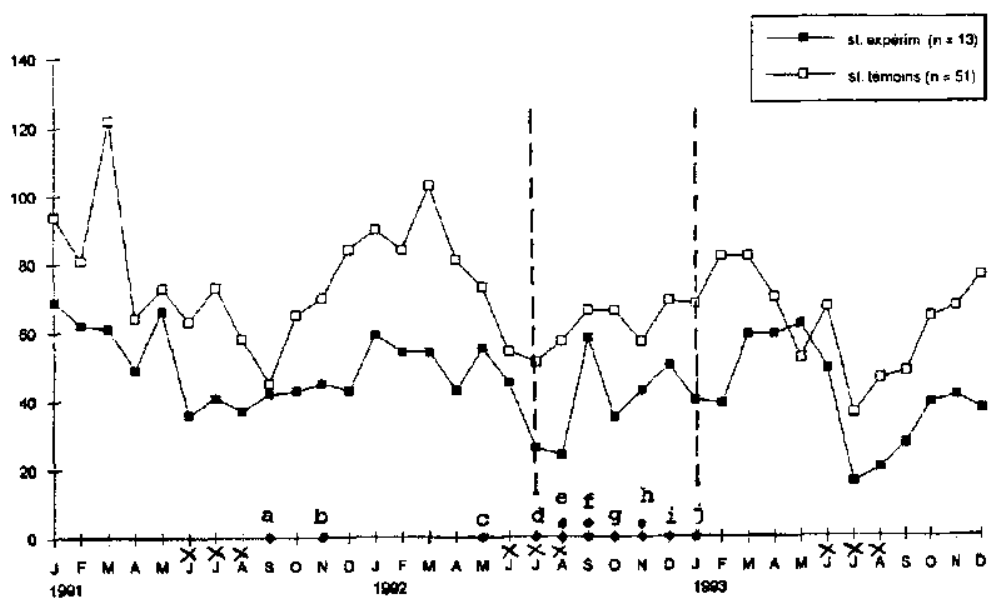

$x x x$ : effets saisonniers

-: mise en service des caméras

a : Lucien-L'Allier (nombre de caméras =6)

b : Lionel-Groulx $(n=10)$

c : île-Sainte-Hélène $(n=12)$

d: Villa-Maria $(n=6)$

e : Du Parc $(n=5)$

Berri-UQAM $(n=20)$

f : $\quad$ McGill $(n=8)$

De Castelnaw $(n=8)$

g: St-Henri $(n=6)$

$h$ : Jean-Talon $(n=12)$

Snowdon $(\mathrm{n}=16)$

i : Plamondon $(\mathrm{n}=8)$

j : Henri-Bourassa $(n=10)$ 
Une première constatation se dégage du graphique 1 : une tendance légère mais constante à la baisse dans le nombre moyen de crimes commis dans le métro entre janvier 1991 et décembre 1993. Cette tendance peut être estimée à la fois pour les stations expérimentales et les stations témoins. Le tableau 2 montre que la tendance à la baisse dans le volume mensuel de délits rapportés à la police est statistiquement significative pour les stations expérimentales et se traduit par une diminution moyenne de 0,52 délit par mois. Comme la série chronologique couvre 36 mois, cela signifie qu'en décembre 1993, le nombre moyen de délits par mois a chuté de 19 délits par rapport à un niveau de base initial (janvier 1991) de 70 environ. Une baisse cumulative importante, par conséquent. Toutefois, la baisse tendancielle est aussi importante pour les stations témoins qui, quant à elles, ont connu une diminution moyenne de 0,66 délit par mois, d'où une baisse cumulative après 36 mois de 24 délits (par rapport à un niveau de base initial de 100 délits environ).

Cette baisse tendancielle du volume mensuel de délits, entre janvier 1991 et décembre 1993, est une bonne nouvelle. La mauvaise nouvelle est que celle-ci se produit à ja fois dans les stations expérimentales et dans les stations témoins. Toutefois, il est possible que la baisse tendancielle soit plus marquée après l'installation des caméras qu'avant, et que cet impact marginal soit noyé ou camouflé dans la diminution générale du niveau de criminalité observée dans le graphique 1 .

\section{Tableau 2}

Impact des caméras sur l'incidence mensuelle de la criminalité, rapportée à la police, dans le métro de Montréal, en contrôlant pour les effets de tendance (janvier 1991 à décembre 1993)

\begin{tabular}{lccc}
\hline & $\begin{array}{c}\text { STATIONS } \\
\text { EXPERIMENTALES }\end{array}$ & $\begin{array}{c}\text { STATIONS } \\
\text { TÉMOINS }\end{array}$ \\
\cline { 2 - 4 } & $\begin{array}{c}\text { Impact } \\
\text { mensuel de } \\
\text { la tendance } \\
\text { (coeff. b) }\end{array}$ & $\begin{array}{c}\text { Impact des } \\
\text { caméras } \\
\text { (coeff. c) }\end{array}$ & $\begin{array}{c}\text { Impact } \\
\text { mensuel de } \\
\text { la tendance } \\
\text { (coeff b) }\end{array}$ \\
\hline $\begin{array}{l}\text { Incidence mensuelle } \\
\text { des délits }\end{array}$ & $-0,52^{*}$ & 7,73 & $-0.66^{*}$ \\
\hline
\end{tabular}

(1) Analyse de régression $Y=a+b X+c Z$ où

$Y=$ incidence des délits par mois entre janvier 1991 et décembre 1993 ;

$X=$ mois successifs durant cette période (l à 36) ;

$Z=$ mois d'installation de la dernière caméra (les mois de janvier 1993 et suivants ayant la valeur de 1 et les mois antérieurs, la valeur de 0 ).

(2) Analyse de régression $Y=a+b X$.

* niveau de signification : $p \leq .05$ 
Pour évaluer l'impact préventif des caméras dans les stations expérimentales, nous avons choisi de comparer l'incidence de la criminalité avant et après l'installation de la dernière station ainsi équipée (en janvier 1993). Les résultats du tableau 2 indiquent que les caméras n'ont pas eu d'impact statistiquement significatif sur l'incidence de la criminalité. Les conclusions de ce diagnostic ne changent pas lorsqu'on contrôle pour les variations mensuelles d'achalandage (taux de délits par million de passagers, cf. annexe 2 , tableau B), lorsqu'on change le point de coupure et que l'on compare les niveaux de criminalité avant et après le début de l'installation des caméras - en juillet 1992 (cf. annexe 2, tableau C) ${ }^{4}$.

\section{IMPACT DIFFÉRENTIEL DES CAMÉRAS PAR TYPE DE DÉLITS}

Il est possible, toutefois, que les caméras aient un impact préventif sur certains types de crimes et aucun impact sur d'autres formes de délits et que cette efficacité spécifique ou locale ne soit pas détectable en analysant l'ensemble des délits. Plusieurs des résultats bénéfiques mentionnés dans la littérature évaluative portent spécifiquement sur les vols qualifiés. Il est raisonnable de penser que les caméras auraient un effet préventif plus marqué pour les délinquants qui préméditent leurs coups que pour ceux qui agissent de manière impulsive. Nous avons donc examiné l'évolution tendancielle de l'incidence mensuelle de plusieurs formes différentes de délîts et l'impact spécifique imputable aux caméras (en choisissant le mois de janvier 1993 comme le début de la période expérimentale).

Les résultats de nos analyses sont présentés au tableau 3. Premier constat : ce sont surtout les vols qualifiés qui ont connu une baisse marquée au cours des trois années de la période d'analyse. Mais cette baisse se produit à la fois dans les stations témoins et les stations expérimentales. La fréquence des méfaits, des vols et des fraudes (y compris les vols à la tire) diminue également dans toutes les stations, mais cette baisse n'est pas significative. Le volume d'agressions, cependant, demeure stationnaire dans les stations témoins, alors qu'il décroît dans les stations expérimentales. Un résultat intéressant qu'on ne peut toutefois imputer à la télé-surveillance : la baisse

4. Nos conclusions sont également les mêmes lorsqu'on désaisonnalise les donnée de la série chronologique ou lorsqu'on introduit une variable pour contrôler les variations saisonnières les plus marquées. Si on essaje de contourner le problème que pase la sélection d'un point de coupure approprié en faisant le dénombrement cumulatif des stations de métro dotées de caméras (0 station entre janvier et août 1991. 1 station en septembre et octobre, 2 stations de novembre 1991 à avril 1992, 3 stations en mai et juin 1992, 4 stations en juillet 1992, 6 en août 1992, ainsi de suite) ou en procédant au décompte cumulatif des différentes caméras installées dans ces stations, on se heurte alors à un problème sérieux de la multicolinéarité. 
n'est pas significative. Pour vérifier si le déclin des vols qualifiés dans les stations expérimentales peut être attribué à la télé-surveillance, nous examinons si cette baisse est plus marquée après qu'avant l'installation des caméras. Nos analyses indiquent que ce n'est pas le cas (aucun impact statistiquement significatif ; par ailleurs, la direction de l'effet observé est, là aussi, contraire à celle que l'on espérait).

Tableau 3

Impact des caméras sur l'incidence mensuelle de la criminalité rapportée à la police, dans le métro de Montréal, par type de délits, et en contrôlant pour les effets de tendance

(janvier 1991 à décembre 1993)

\begin{tabular}{lcccc}
\hline Incidence mensuelle & \multicolumn{2}{c}{$\begin{array}{c}\text { STATIONS } \\
\text { EXPÉRIMENTALES }\end{array}$} & $\begin{array}{c}\text { STATIONS } \\
\text { TÉMOINS }\end{array}$ \\
\cline { 2 - 3 } & $\begin{array}{c}\text { Impact } \\
\text { mensuel de } \\
\text { la tendance } \\
\text { (coeff. b) }\end{array}$ & $\begin{array}{c}\text { Impact des } \\
\text { caméras } \\
\text { (coeff. c) }\end{array}$ & & $\begin{array}{c}\text { Impact } \\
\text { mensuel de } \\
\text { la tendance } \\
\text { (coeff b) }\end{array}$ \\
\hline Vols qualifiés & $-0,19 *$ & & $-0,29^{*}$ \\
Agressions, voies de fait & $-0,09$ & 1,17 & 0,05 \\
Vols à la tire & $-0,14$ & 0,48 & $-0,11$ \\
Vols, fraudes & $-0,05$ & 8,53 & $-0,19 *$ \\
Méfaits & $-0,01$ & $-0,96$ & $-0,02$ \\
Autres infractions & $-0,04$ & $-0,81$ & $-0,11$ \\
\hline
\end{tabular}

(1) Analyse de régression $Y=a+b X+c Z$ où

$Y=$ incidence des délits par mois entre janvier 1991 et décembre 1993 ;

$X=$ mois successifs durant cette période ( 1 à 36);

$Z=$ mois d'installation de la dernière caméra (les mois de janvier 1993 et suivants ayant la valeur de 1 et les mois antérieurs, la valeur de 0 ).

(2) Analyse de régression $Y=a+b X$.

* niveau de signification : $p \leq .05$

\section{LE NOMBRE DE CAMÉRAS ET LA SÉLECTION DES SITES DE LEUR EMPLACEMENT}

Il est possible que les caméras de télévision aient des effets préventifs uniquement lorsque certaines conditions sont réunies. Par exemple, les caméras pourraient avoir un effet préventif, mais seulement sur les délits commis dans l'aire que celles-ci balaient. Par ailleurs, la criminalité est inégalement distribuée dans l'espace intérieur de chaque station, certains endroits ou secteurs étant plus à risque que d'autres. Pour tenir compte de cette complication, de nouvelles analyses sont nécessaires.

Nous avons brièvement examiné la topographie de la criminalité dans les stations expérimentales et vérifié la répartition correspondante des 
caméras. La partie gauche du tableau 4 indique qu'en nombre absolu, le quart environ des délits se produisent dans les wagons et que le reste des délits se distribuent de manière à peu près égale dans les corridors, les quais, les édicules (entrées/sorties), les loges des changeurs et les aires connexes (dépanneurs, aires d'attente extérieures). Compte tenu des limites de cette étude, nous n'avons pas calculé une donnée qui a pourtant une importance stratégique pour tout projet de télé-surveillance : la densité spatiale des crimes commis. Une caméra est d'autant plus efficace, en termes préventifs, que le ratio entre les délits qui s'y produisent et la superficie du secteur où ils sont commis est élevé. À nombre égal de caméras, il est préférable de cibler de petites superficies à risque que des grandes.

Quoi qu'il en soit, un résultat important du tableau 4 est le suivant : $60 \%$ des caméras ont été installées dans un secteur du métro où ne se commettent que $16 \%$ des délits rapportés à la police. À l'inverse, $27 \%$ des délits se produisent dans les wagons où aucune caméra n'a été installée. Par ailleurs, on peut penser que certains secteurs du métro sont moins bien surveillés que d'autres (en ce sens que le nombre de caméras par mètre carré de corridor ou d'escalier est probablement plus petit que le nombre de caméras par mètre caré de quai).

\section{Tableau 4}

Distribution de la criminalité et des caméras pour les stations expérimentales du métro de Montréal, en fonction du type d'endroits y correspondant (juillet 1991 à juin 1992)

\begin{tabular}{lccccc}
\hline TYPE D'ENDROITS & \multicolumn{2}{c}{ CRIMINALITÉ } & & \multicolumn{2}{c}{ CAMÉRAS $^{2}$} \\
\cline { 2 - 3 } \cline { 5 - 6 } & $\mathrm{N}$ & $\%$ & & $\mathrm{~N}$ & $\%$ \\
\hline Véhicule & 150 & 26,7 & & 0 & 0,0 \\
Corridor, escalier, mezzanine & 95 & 16,9 & & 33 & 26,0 \\
Quai & 91 & 16,2 & & 74 & 58,3 \\
Édicule (entrée/sortie) & 81 & 14,4 & & 18 & $1.1,2$ \\
Loge des changeurs & 75 & 13,4 & & $0^{3}$ & 0,0 \\
Autres endroits' & 69 & 12,3 & & 2 & 1,6 \\
\hline Total & 561 & 99,9 & & 127 & 100,1 \\
\hline
\end{tabular}

Valeur manquante var. crimes : 1

1. Dépanneur. local STCUM, aire d'attente extérieure, etc.

2. Un certain nombre de caméras filment deux endroits en même temps, par exemple une partie de l'escalier et de l'édicule ; le choix de l'emplacement dans ces cas est arbitraire.

3. Les caméras aux loges des changeurs ont été installées après la période expérimentale.

Il est donc possible que nos analyses antérieures aient été partiellement biaisées. Comme le quart de la criminalité totale rapportée à la police se 
produit dans les wagons, nous ne devrions pas évaluer l'impact préventif des caméras de télévision en incluant dans nos analyses l'ensemble des délits que celles-ci ne pouvaient pas, de toutes façons, prévenir. Par ailleurs, il est également possible que les caméras de télévision aient été très efficaces dans la prévention des délits commis sur les quais, mais que cette efficacité ne puisse être détectée en analysant l'ensemble des délits du métro.

Nous avons donc recommencé nos analyses en distinguant cette fois la criminalité commise sur les quais des autres délits et en éliminant des données utilisées pour évaluer l'impact de l'expérience-pilote les délits commis dans les wagons. Le tableau 5 présente les résultats de ces analyses. Première constatation : le seul secteur du métro où la criminalité n'a pas connu une baisse tendancielle est celui des quais. Cela est vrai à la fois des stations témoins et des stations expérimentales où pourtant $60 \%$ des caméras de l'expérience-pilote ont été installées.

\section{Tablean 5}

Impact des caméras sur l'incidence mensuelle de la criminalité rapportée à la police, dans le métro de Montréal, par type d'endroits dans la station et en contrôlant pour les effets de tendance (janvier 1991 à décembre 1993)

\begin{tabular}{|c|c|c|c|}
\hline \multirow[t]{2}{*}{ Incidence mensuelle } & \multicolumn{2}{|c|}{$\begin{array}{c}\text { STATIONS } \\
\text { EXPÉRIMENTALES }\end{array}$} & \multirow{2}{*}{ 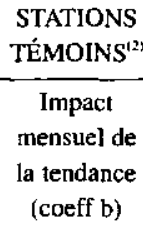 } \\
\hline & $\begin{array}{c}\text { Impact } \\
\text { mensuel de } \\
\text { la tendance } \\
\text { (coeff. b) }\end{array}$ & $\begin{array}{l}\text { Impact des } \\
\text { caméras } \\
\text { (coeff. c) }\end{array}$ & \\
\hline \multicolumn{4}{|l|}{ Sur les quais } \\
\hline $\begin{array}{l}\text { seulement } \\
\text { Partout, sauf dans }\end{array}$ & $-0,01$ & 4.26 & -0.11 \\
\hline $\begin{array}{l}\text { les wagons } \\
\text { Partout, sauf sur } \\
\text { les quais et dans }\end{array}$ & $-0,41^{*}$ & 3,23 & $-0,71^{*}$ \\
\hline les wagons & $-0,40^{*}$ & $-1,15$ & $-0,60^{*}$ \\
\hline
\end{tabular}

(1) Analyse de régression $Y=a+b X+c Z$ où

$Y=$ incidence des délits par mois entre janvier 1991 et décembre 1993 ;

$X=$ mois successifs durant cette période ( 1 à 36 ) ;

$Z=$ mois d'installation de la dernière caméra (les mois de janvier 1993 et suivants ayant la valeur de 1 et les mois antérieurs, la valeur de 0 ).

(2) Analyse de régression $Y=a+b X$.

* niveau de signification : $\mathrm{p} \leq .05$

Deuxième constatation : on observe (dernière rangée du tableau 5) une baisse tendancielle des délits qui sont commis dans les corridors, les édicules, 
les aires d'attente et les mezzanines, à la fois dans les stations témoins et les stations expérimentales. Et pour la première fois, on observe un impact préventif des caméras de télévision. Malheureusement, cet effet préventif n'est pas statistiquement significatif.

\section{EFFET DE SEUIL ET ANALYSE D'UNE STATION EXPÉRI- MENTALE AYANT LE VOLUME MENSUEL DE DÉLITS LE PLUS ÉLEVÉ DU RÉSEAU}

Le nombre mensuel de délits par station de métro est relativement faible pour fins d'analyse statistique. Il est possible qu'en deçà d'un certain volume, les délits se commettent à une fréquence trop sporadique pour qu'on puisse les prévenir. En d'autres termes, les projets de prévention auraient d'autant plus de chances d'avoir des effets bénéfiques que la masse de délits à prévenir est importante. Comme plusieurs des stations expérimentales (ainsi que nous le notions au début de cette étude) n'ont pas été choisies en fonction de leur volume élevé de crimes (ou du niveau des risques individuels de victimisation), on ne peut pas éliminer la possibilité que l'impact des caméras ait varié d'une station de métro à l'autre (tout dépendant de leur volume respectif de criminalité). Si notre hypothèse est vraie, on devrait s'attendre à ce que la station expérimentale où le volume mensuel de délits est le plus élevé de tout le circuit (même si les risques individuels de victimisation y sont en réalité relativement modérés) — la station Berri-UQAM - soit également la station où l'on devrait observer un effet préventif maximal de la télésurveillance (cf. graphique 2).

Mais cette dernière hypothèse ne résiste pas, elle non plus, à l'épreuve des faits. À Berri, comme dans les autres stations, le niveau global de la criminalité ne semble pas avoir été affecté par la mise en place des caméras : leur impact, si impact il y a, s'exerce dans la mauvaise direction (les crimes augmentent après leur installation) et n'est pas, de toute façon, statistiquement significatif. La situation ne change pas lorsqu'on adopte une mesure d'incidence de la criminalité qui tienne compte des risques individuels de victimisation (taux de délits par million de passagers), même si avec cette nouvelle mesure, la direction des effets imputables aux caméras s'exerce cette fois dans le bon sens (le taux de délits baisse suite à leur installation, bien que cette baisse ne soit pas statistiquement significative). Si l'on examine séparément les diverses formes de délinquance, les caméras semblent avoir diminué le volume mensuel des vols qualifiés et des agressions, mais cette diminution, à nouveau, n'est pas statistiquement significative. L'analyse particulière de cette station de métro aboutit à des résultats trop modestes pour remettre en cause notre précédent constat d'échec. (On trouvera en annexe 3 le détail de ces analyses.) 


\section{Graphique 2}

Incidence mensuelle de la criminalité rapportée à la police, dans la station Berri-UQAM : l'ensemble de la criminalité et les agressions, voies de fait et vols qualifiés (janvier 1991 à décembre 1993)

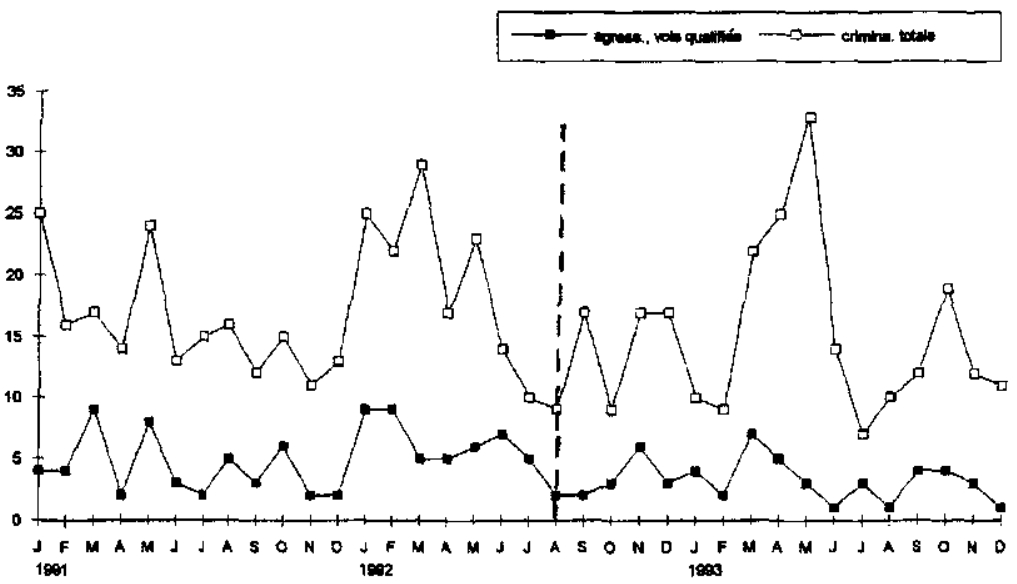

\section{CONCLUSION}

Même si les stratégies de prévention situationnelle de la criminalité obtiennent généralement des résultats encourageants et mêrne si l'impact préventif des systèmes de télé-surveillance dans les métros est bien documenté dans certains réseaux (celui de Londres, par exemple), l'expériencepilote réalisée par la STCUM apparaît néanmoins, sur la base des données disponibles, comme un échec. Il est vrai que la criminalité rapportée à la police a connu une tendance à la baisse fort significative entre janvier 1991 et décembre 1993, mais cette tendance vaut à la fois pour les stations de métro où aucune caméra n'a été installée et pour les stations de métro équipées de telles caméras.

Le problème, dès lors, est le suivant : pourquoi un tel échec, alors qu'on pouvait raisonnablement espérer, sur la base des expériences antérieures, de bien meilleurs résultats ? Nous proposons ici un certain nombre d'éléments de réponse.

\section{La concentration des ressources}

« La grande leçon des évaluations de plus de 200 projets de prévention dans les Pays-Bas est que nous ne pouvons pas obtenir de résultat si l'intervention manque d'intensité " (Cusson et coll., 1994, p. 40.) Cette intensité 
peut être acquise par la concentration des moyens sur un point précis ou un type spécifique de délits. Dans l'expérience-pilote faite à Montréal, les caméras de télévision ont été saupoudrées dans 13 stations de métro. Et dans chaque station de métro, la distribution des caméras s'est faite de manière largement indépendante de la topographie réelle de la criminalité. Il aurait été plus efficace de concentrer les ressources dans moins de stations mais de cibler celles qui présentaient clairement un niveau de criminalité préoccupant. Il aurait été également plus efficace de concentrer les caméras (pour chacune des stations expérimentales) dans les zones où la densité et le volume de délits, d'une part, et les risques de victimisation, d'autre part, étaient les plus élevés.

\section{La crédibilité des moyens dissuasifs utilisés.}

Un projet de prévention vise, en dernière analyse, à convaincre les délinquants potentiels d'abandonner leurs projets de commettre des délits parce qu'ils estiment que les risques qu'ils courent sont désormais trop élevés. Comme la plupart des délinquants sont généralement opportunistes et que leur motivation initiale est souvent d'intensité modérée ou faible, les programmes de prévention situationnelle obtiennent généralement de bons résultats. Encore faut-i] que l'augmentation des risques soit réelle pour que la stratégie de prévention soit crédible. Il ne suffit pas, en somme, d'installer des caméras pour qu'automatiquement les délinquants potentiels modifient leurs comportements. Encore faut-il que ces caméras produisent des effets ultérieurs tangibles qui convainquent, initialement, un petit nombre de délinquants, qui par la suite transmettront de manière informelle l'information à leurs pairs. Or, dans l'expérience-pilote que nous avons examinée dans cette étude, la crédibilité dissuasive des caméras ne va pas de soi.

D'une part, les caméras du métro tournent à vide, en ce sens que personne ne les regarde pour diagnostiquer un incident. Même lorsque les moniteurs sont placés devant les loges des changeurs, ces derniers n'ont pas pour tâche explicite de surveiller ces moniteurs. En fait, lorsqu'une agression est commise, c'est généralement la victime qui avertit le changeur, et ce n'est qu'après coup qu'on visionne les bandes pour fins d'enquête policière (dans le métro de Londres, par contre, l'exploitation des caméras est beaucoup plus systématique ; Webb et Laycock, 1992).

D'autre part, la crédibilité de la télé-surveillance est proportionnelle à son utilité subséquente pour fins d'enquête policière, c'est-à-dire au pouvoir de résolution et à la précision des images. Or, les caméras installées dans le projet-pilote produisent des images en noir et blanc (les images en couleurs sont beaucoup plus informatives ; Garrett et Goldstein, 1994), ne sont pas munies d'un zoom (comme à Londres, encore une fois, Webb et Laycock. 
1992) et sont installées dans un boîtier fixe (plutôt que mobile). De plus, en raison du petit nombre de caméras par station et de leur dispersion territoriale, la distance moyenne entre la cible et la caméra est généralement trop élevée pour permettre une identification suffisante pour fins d'enquête policière ou de preuve devant les tribunaux. Résultat : des images imprécises, des bandes de visionnement rarement utiles pour les policiers et, par conséquent, rarement mentionnées comme pièce à conviction dans les médias.

\section{La publicité comme mesure connexe}

L'expérience-pilote dans le métro de Montréal s'est faite très discrètement, avec le moins de publicité possible. Dans les métros de Londres et de Hong Kong, par contre, les caméras ont été installées à grand renfort de publicité. Il ne s'agissait pas seulement de rassurer les usagers, mais de communiquer un message aux délinquants potentiels. Bien qu'il soit erroné de croire que la publicité (même une publicité de haute qualité) puisse se substituer à un programme de prévention, elle constitue, dans certains cas, une mesure complémentaire qui permet de renforcer les effets préventifs souhaités en accélérant la diffusion d'un message initialement bien-fondé et crédible (concernant, par exemple, les risques de détection encourus par les délinquants potentiels).

Dans le cas de Montréal, il n'aurait pas été opportun de se livrer à cet exercice de publicisation. D'une part, la crédibilité dissuasive du système de surveillance mis en place était incertaine (comme on peut s'en rendre compte, aujourd'hui, en examinant les statistiques criminelles pertinentes). D'autre part, les objectifs poursuivis par l'expérience-pilote étaient trop nombreux. Outre le désir de prévenir la criminalité, on souhaitait également mieux évaluer les déplacements de foule (d'où l'installation des caméras sur les quais et dans les stations de correspondance). On était aussi préoccupé par les résultats d'un sondage sur le sentiment d'insécurité des usagers du métro (réalisé en 1989 par la firme Léger et Léger), et ce sondage a incité les concepteurs de l'expérience à installer des caméras dans des stations où les crimes étaient rares, mais où le sentiment d'insécurité des usagers semblait très marqué. De là le grand nombre de stations expérimentales où le volume de criminalité est faible et les risques de victimisation minimes ${ }^{s}$.

Pour finir, quelques remarques concernant la méthodologie évaluative utilisée dans cette étude. Lorsqu'un programme de prévention de la criminalité

5. Sur les difficultés que l'on rencontre en donnant aux projets de prévention une fonction subjective - diminuer le sentiment d'insécurité des victimes potentielles -, plutôt que comportementale - diminuer la fréquence des délits susceptibles d'être commis par des délinquants potentiels —, voir Tremblay et coll., 1993 ; Cusson et coll., 1994, p. 34. 
réussit, la tentation est grande d'attribuer le succès obtenu aux moyens utilisés. Un bon plan d'évaluation reconnaît au départ que l'impact observé peut être la résultante de toute une série de facteurs extrinsèques aux ressources mobilisées par le programme et qu'il est nécessaire d'éliminer l'une après l'autre les explications rivales susceptibles d'être opposées au diagnostic de succès. Lorsqu'un programme de prévention échoue, par contre, la tentation est de tirer un trait sur le programme lui-même et d'essayer quelque chose de «nouveau ». Dans cette étude, l'échec observé ne doit pas être interprété comme un échec de la télé-surveillance, mais plutôt comme un raté de sa mise en œuvre. Le problème principal ne réside pas dans les caméras elles-mêmes ou encore dans la qualité des images qu'elles produisent (bien qu'il s'agisse d'un problème réel), mais dans la manière dont elles ont été utilisées, les objectifs (trop nombreux) qu'on cherchait à leur faire atteindre et l'absence de mesures complémentaires qui étaient capables de rendre la télé-surveillance crédible auprès des délinquants potentiels.

Dans une étude récente sur l'évaluation des projets de prévention du crime, on notait que leur planification implique cinq opérations distinctes : « $1^{\circ}$ identifier et analyser le problème criminel à contrer; $2^{\circ}$ se fixer des buts clairs, spécifiques et mesurables ; $3^{\circ}$ choisir des moyens qui auront des chances d'agir sur les causes rapprochées du problème criminel ciblé ; $4^{\circ}$ obtenir une concentration suffisante des moyens ; $5^{\circ}$ mobiliser les partenaires » (Cusson et coll., 1994, p. 23). Des cinq opérations, seule la troisième a été mise en ceuvre dans l'expérience-pilote de la STCUM.

\section{BIBLIOGRAPHIE}

BURRows J. N. (1980), « Closed Circuit Television and Crime on the London Underground », in R. V. Clarke et P. Mayhew (eds), Designing out Crime, Londres: Home Office.

COOK T. D. et CAMPBELL D. T. (1979), Quasi-Experimentation: Design and Analysis Issues for Field Settings, Boston: Houghton Mifflin.

CUSSON M., TREMBLAY P., BIRON L., OUIMET M., et GRANDMAISON R. (1994), La Planification et l'évaluation de projets en prévention du crime. Québec, Ministère de la Sécurité publique, Gouvernement du Québec.

FORTIN A. (1982), « Plans de recherche quasi expérimentaux », in M. Robert (éd), Fondements et étapes de la recherche scientifique en psychologie, Montréal, Chenelière et Stanké, pp. 119-132.

GARRETT T. et GOLDSTEIN E. (1994), « Color Yields More Useful Videotapes », Canadian Security. The Journal of Protection \& Communications, vol. 16, nº 3, pp. 18-19.

GAYLORD M. S. et GALLIHER J. F. (1991), "Riding the Underground Dragon : Crime Control and Public Order on Hong Kong's Mass Transit Railway », British Joumal of Criminology, vol. 31, n ${ }^{\circ}$, pp. 15-26.

LEGGER \& LÉGER (1989), Les Perceptions des Montréalais à l'égard de la violence dans le métro et les autobus, Montréal. 
TREMBLAY P., CORDEAU G. et KACZOROWSKI J. (1993), « La peur du crime et ses paradoxes : cartes mentales, écologie criminelle et sentiment d'insécurité », Revue canadienne de criminologie, vol. $35, \mathrm{n}^{\circ} 1$, pp. 1-18.

WEBB B. et LAYCOCK G. (1992), Reducing Crime on the London Underground. An Evaluation of Three Pilot Projects, Crime Prevention Unit, Paper no. 30, Londres, Home Office.

\section{Annexe 1}

Tableau A : Nombre et taux de crimes (par million de passagers) par station dans le métro de Montréal (juillet 1991 à juin 1992)

\begin{tabular}{|c|c|c|}
\hline $\begin{array}{l}\text { Numéro et nom } \\
\text { de la station }\end{array}$ & $\begin{array}{l}\text { Nombre de } \\
\text { crimes }\end{array}$ & $\begin{array}{l}\text { Taux de } \\
\text { crimes }\end{array}$ \\
\hline 1 H.-Bourassa* & $57 * *$ & 6,56 \\
\hline 2 Sauvé & 15 & 3,71 \\
\hline 3 Crémazie & 16 & 4,23 \\
\hline 4 Jarry & 23 & 6,63 \\
\hline 5 J.-Talon* & $55^{* *}$ & 10.37 \\
\hline 6. Beaubien & 14 & 4,04 \\
\hline 7 Rosemont & 10 & 2,90 \\
\hline 8 Laurier & 29 & 7,10 \\
\hline 9 Mont-Royal & $35^{* *}$ & 8,87 \\
\hline 10 Sherbrooke & $47 * *$ & $11,30^{* * *}$ \\
\hline 1) Berri-UQAM* & $212 * *$ & $22,38 * *$ \\
\hline 12 Champ-de-Mars & 5 & 2,84 \\
\hline $13 \mathrm{Pl}$. d'Armes & 10 & 2,24 \\
\hline 14 Sq Victoria & 10 & 2,01 \\
\hline 15 Bonaventure & 8 & 2.33 \\
\hline 16 L.-L'Allier* & 5 & 8.54 \\
\hline 17 G.-Vanier & 24 & $37,98 * *$ \\
\hline 18 Charlevoix & 17 & $14.03 * *$ \\
\hline 19 Lasalle & 7 & 5,10 \\
\hline 20 De L'Église & 19 & $11,16 * *$ \\
\hline 21 Verdun & 16 & $12,80^{* *}$ \\
\hline 22 Jolicoeur & 14 & 8,22 \\
\hline 23 Monk & 11 & 11.15 \\
\hline 24 Angrignon & 21 & 4,86 \\
\hline 25 Île-Ste-Hélène* & 2 & 2,82 \\
\hline 26 Longueuil & ND & ND \\
\hline 27 Côte-Vertu & $38 * *$ & 7,84 \\
\hline 28 Du Collège & 7 & 3,06 \\
\hline 29 De La Savane & 4 & 6.29 \\
\hline 30 Namur & 7 & 4.85 \\
\hline 31 Plamondon* & $30 * *$ & 8,46 \\
\hline 32 Côte-Ste-Catherine & 11 & 6,49 \\
\hline 33 Snowdon* & $30 * *$ & $12,24 * *$ \\
\hline 34 Villa-Maria* & 13 & 4,78 \\
\hline 35 Vendôme & 21 & 4,66 \\
\hline
\end{tabular}


Annexe 1 (suite)

\begin{tabular}{|c|c|c|}
\hline $\begin{array}{l}\text { Numéro et nom } \\
\text { de la station }\end{array}$ & $\begin{array}{l}\text { Nombre de } \\
\text { crimes }\end{array}$ & $\begin{array}{l}\text { Taux de } \\
\text { crimes }\end{array}$ \\
\hline 36 Pl. St-Henri* & 29 & $16,14^{* *}$ \\
\hline 37 Lionel-Groulx* & $57^{* *}$ & $16,03 * *$ \\
\hline 38 Atwater & $38 * *$ & $31,36^{* *}$ \\
\hline 39 Guy-Concordia & $30^{* *}$ & 4.55 \\
\hline 40 Peel & $33 * *$ & 5.00 \\
\hline 41 McGill* & $50^{* *}$ & 4,10 \\
\hline 42 Place-des-Arts & 21 & 3,44 \\
\hline 43 St-Laurent & $31^{* *}$ & $15,48 * *$ \\
\hline 44 Beaudry & 29 & $27,59 * *$ \\
\hline 45 Papineau & 21 & 6,88 \\
\hline 46 Frontenac & 5 & 2,30 \\
\hline 47 Préfontaine & 8 & 8,92 \\
\hline 48 Joliette & 23 & 10,87 \\
\hline 49 Pie-IX & 17 & 3,80 \\
\hline 50 Viau & 13 & 6,87 \\
\hline 51 L'Assomption & 10 & $13,59^{* *}$ \\
\hline 52 Cadillac & 21 & 9,22 \\
\hline 53 Langelier & 17 & 7,48 \\
\hline 54 Radisson & 26 & 8,74 \\
\hline 55 H.-Beaugrand & $46^{* *}$ & 10,28 \\
\hline 56 Côte-des-Neiges & 10 & 4,22 \\
\hline 57 Université de Mtl & 0 & 0,00 \\
\hline 58 É.-Montpetit & 2 & 2,08 \\
\hline 59 Outremont & 6 & 5,94 \\
\hline 60 Acadie & 3 & 4,94 \\
\hline 61 Parc* & 14 & 6.58 \\
\hline 62 De Castelnau* & 8 & 7,15 \\
\hline 63 Fabre & 9 & 5,86 \\
\hline 64 D'Iberville & 1 & 1,14 \\
\hline 65 St-Michel & 21 & 7,86 \\
\hline Total & 1442 & $7,3,5$ \\
\hline
\end{tabular}

* Les 13 stations sélectionnées pour être équipées du système de télé-surveillance par caméras.

** Les 13 résultats les plus élevés aux colonnes « nombre de crimes » et « taux de crimes ». 


\section{Annexe 2}

Tableau B : Impact des caméras sur le taux mensuel de crimes rapportés à la police, dans le métro de Montréal, en contrôlant pour les effets de tendance (janvier 1991 à décembre 1993)

\begin{tabular}{lcc}
\hline & \multicolumn{1}{c}{ STATIONS EXPÉRIMENTALES $^{(1)}$} \\
\cline { 2 - 3 } & $\begin{array}{c}\text { TAUX DE CRIMES } \\
\text { (par million de passagers) }\end{array}$ \\
\hline $\begin{array}{l}\text { Impact mensuel de la } \\
\text { tendance (coeff. b) }\end{array}$ & $\begin{array}{c}\text { Impact des caméras } \\
\text { (coeff. c) }\end{array}$ \\
\hline \begin{tabular}{l} 
des délits \\
\hline
\end{tabular}
\end{tabular}

(1) Analyse de régression $Y=a+b X+c Z$ où

$Y=$ incidence des délits par mois entre janvier 1991 et décembre 1993 ;

$X=$ mois successifs durant cette période ( 1 à 36 ) ;

$Z=$ mois d'installation de la dernière caméra (les mois de janvier 1993 et suivants ayant la valeur de 1 et les mois antérieurs, la valeur de 0 ).

* niveau de signification : $\mathrm{p} \leq 0,05$.

Tableau C : Impact des caméras sur l'incidence mensuelle de la criminalité rapportée à la police, dans le métro de Montréal, en choisissant le mois de juillet comme le début de la période expérimentale (janvier 1991 à décembre 1993)

\begin{tabular}{|c|c|c|}
\hline & \multicolumn{2}{|c|}{ STATIONS EXPÉRIMENTALES ${ }^{\prime \prime}$} \\
\hline & \multicolumn{2}{|c|}{ NOMBRES ABSOLUS } \\
\hline & $\begin{array}{c}\text { Impact mensuel de la } \\
\text { tendance (coeff. b) }\end{array}$ & $\begin{array}{c}\text { Impact des caméras } \\
\text { (coeff. c) }\end{array}$ \\
\hline $\begin{array}{l}\text { Incidence mensuelle } \\
\text { des délits }\end{array}$ & $-0.52^{*}$ & $-2,43$ \\
\hline
\end{tabular}

(1) Analyse de régression $Y=a+b X+c Z$ où

$Y=$ incidence des délits par mois entre janvier 1991 et décembre 1993 ;

$X=$ mois successifs durant cette période (1 à 36) ;

$Z$ = mois correspondant au début de la période intensive d'installation de caméras (les mois de juillet 1992 et suivants ayant la valeur de 1 et les mois antérieurs, la valeur de 0 ).

* niveau de signification : $\mathrm{p} \leq 0,05$. 


\section{Annexe 3}

Tableau D : Impact des caméras sur l'incidence mensuelle de la criminalité rapportée à la police, dans la station Berri-UQAM, en contrôlant pour les effets de tendance (janvier 1991 à décembre 1993)

\begin{tabular}{lcccc}
\hline & \multicolumn{3}{c}{ STATION BERRI-UQAM $^{(1)}$} \\
\cline { 2 - 5 } & $\begin{array}{c}\text { Impact } \\
\text { mensuel } \\
\text { (coeff. b) }\end{array}$ & $\begin{array}{c}\text { Impact } \\
\text { des } \\
\text { caméras } \\
\text { (coeff. c) }\end{array}$ & $\begin{array}{c}\text { Impact } \\
\text { mensuel } \\
\text { (coeff. b) }\end{array}$ & $\begin{array}{c}\text { Impact } \\
\text { des } \\
\text { caméras } \\
\text { (coeff. c) }\end{array}$ \\
\hline $\begin{array}{l}\text { Incidence mensuelle } \\
\text { des délits }\end{array}$ & $-0,11$ & 1,80 & $-0,23$ & 4,09 \\
\hline
\end{tabular}

(1) Analyse de régression $Y=a+b X+c Z$ où

$Y=$ incidence des délits par mois entre janviex 1991 et décembre 1993 ;

$X=$ mois successifs durant cette période (l à 36) :

$Z$ = mois d'installation de la dernière caméra (les mois de janviers 1993 et suivants ayant la valeur de 1 et les mois antérieurs, la valeur de 0 ).

* niveau de signification : $\mathrm{p} \leq 0,05$. 